sumptions, social context and effects of laws and to stimulate reforms in the legal system and processes of implementing development policies."

Die Realisierung eines solchen - ohnehin nur langfristig $\mathrm{zu}$ denkenden - Programms muß bei der Neubestimmung der Ziele ansetzen und neue Prozesse und Institutionen einer systematischen Planung initiieren. Dazu gehört vor allem eine exakte Bestandsaufnahme der jeweiligen nationalen Rechtskultur, der Berufsmöglichkeiten und des Bedarfs an speziellen Qualifikationen. An anderer Stelle heißt es, es müßten zunächst die "Strukturen für eine effektivere Planung organisiert" werden. Also nicht ein Plan oder mehrere Pläne sind das Ziel, sondern - bescheidener und realistischer - die Schaffung eines Klimas für Planung und Entwicklung überhaupt.

Diese Überlegungen zur Reformplanung gehen über in Problemskizzen zu Einzelfragen. Hier wird zwischen einem möglichen Programm für ein Grundstudium der Rechtswissenschaft (First Degree in Law) und Spezialprogrammen (Aufbaustudium für Spezialisten, Ausbildung von "paraprofessionals“ und Verbreitung von juristischer Information) unterschieden; Probleme der rechtswissenschaftlichen Literatur und Forschung, der Ausbildung von Rechtslehrern und der Finanzierung von Law Schools bilden den Abschluß. Auch in diesem Rahmen finden sich Feststellungen, die in gleicher Weise auf die „westlichen“ Ausbildungssysteme zutreffen, z. B. eine knappe aber tiefdringende Kritik des Prüfungswesens.

Es ist nicht möglich, die jahrelange Arbeit der Expertengruppe, die außer eigenen Beobachtungen eine Vielzahl von Quellen herangezogen hat, auf ihre Richtigkeit zu überprüfen. Alles spricht dafür, daß die Analyse stimmt. Die rechtspolitischen und didaktischen Probleme sind treffend dargestellt, und die Reformvorschläge sind wohlbegründet. Aus der Erfahrung der deutschen Ausbildungsreform heraus würde man freilich die Entwicklungsfähigkeit der Rechtswissenschaft etwas skeptischer einschätzen. Wenn die ILC-Gutachter die Handlungsmöglichkeiten von Juristen etwa in bezug auf die landwirtschaftliche Entwicklung oder die staatlichen Unternehmen stark hervorheben, so mag dies aus den Verhältnissen der Entwicklungsländer heraus begründet sein; als deutscher Jurist ist man jedoch fast versucht, hier Tendenzen zu erkennen, die in späterer Zukunft auf ein Juristenmonopol hinauslaufen könnten - was sicher nicht im Sinne der Autoren wäre. Wer die Schwierigkeiten kennt, mit denen Ausbildungsreformen in hochentwickelten Staaten zu kämpfen haben, wird auch die Aussichten weitreichender Neuorientierung in Entwicklungsländern nicht ganz so günstig beurteilen wie die Verfasser dieser Schrift. Aber wenn die Entwicklungsländer große Anstrengungen in der hier aufgezeigten Richtung unternehmen, mag es durchaus sein, daß sie eines Tages ihre früheren Lehrmeister eines Besseren belehren.

Hans Peter Bull

\title{
Werner Morvay
}

Souveränitätsübergang und Rechtskontinuität im Britischen Commonwealth. Ein Beitrag zur Lehre von der Staatensukzession

(Beiträge zum ausländischen öffentlichen Recht und Völkerrecht Band 65) Springer Verlag Berlin Heidelberg New York 1974, VIII, 116 S.

Das angezeigte Buch ist einer der ersten Versuche in der bundesdeutschen Literatur, einige juristische Probleme im Zusammenhang zu beleuchten, die die Auf- 
lösung des britischen Kolonialreichs mit sich brachte. Der Verfasser dieses Buches erhebt dabei allerdings keinen ähnlich umfassenden Anspruch wie Albert Bleckmann, der 1970 die Auflösung des französischen Kolonialreichs untersucht hat ${ }^{1}$; vielmehr beschränkt er sich darauf, einige spezifische Probleme der Staatensukzession im Bereich des Commonwealth zu diskutieren. Die postkoloniale Rechtsentwicklung in den jungen Commonwealth-Staaten wird nur im letzten Kapitel kurz angesprochen. Die beträchtliche Fülle an Detailinformationen wäre durch die Anfügung eines Sachregisters und eines Registers der erwähnten Gerichtsentscheidungen zugänglicher geworden.

Das Commonwealth bestand bereits im Jahre 1973 aus zweiunddreißig Vollmitgliedern und einem Mitglied mit Sonderstatus. Der Liste des Verfassers (S. 4) sind hinzuzufügen: Nauru (Sonderstatus 1968) und West-Samoa (Vollmitglied 1970). Weitere neue Mitglieder sind Grenada (1974) sowie Papua-Neuguinea (1975), und am 28. 6. 1976 wird die Inselgruppe der Seychellen dem Commonwealth als fünfunddreißigstes Vollmitglied beitreten.

Der Autor gibt im ersten Kapitel einen kurzen historischen Überblick über die Verselbständigung der ehemaligen Dominions von der Kolonialmacht sowie über die Unabhängigkeitsgewährungen nach dem zweiten Weltkrieg. Gerade der Emanzipationsprozeß der Dominions, der eher einer "Staatenevolution“ als einer Staatensukzession gleicht, verdeutlicht die britische Dekolonisationsmethode der „verfassungsrechtlichen Dezentralisierung" (S. 27). Anders als Frankreich hat Großbritannien nur in wenigen Fällen den völkerrechtlichen Vertrag als Instrument der Entkolonialisierung eingesetzt (S. 92 ff.).

Bei der Erörterung der internen Struktur der Dominions streift der Autor auch kurz die wichtigsten Organisationsprinzipien, die die Briten ihren Kolonien schon früh zur Nachahmung empfahlen (S. 13 f.), das, was S. A. de Smith als ,Westminster export model' bezeichnet hat, nämlich die Grundsätze der ,representative legislature' und des ,responsible government'. Dies ist eine der wenigen Passagen des Buches, in denen trotz der engen Fragestellung auch inhaltliche Probleme der Verfassungsrechtstradition im Commonwealth angeschnitten werden. Eingehend wird dagegen beschrieben, wie die Kontrollmechanismen über das Gesetzgebungsrecht der Dominions, die ,reservation of bills' und die ,disallowance of laws' - „Instrumente der Fremdherrschaft" (S. 18) - allmählich durch Verfassungskonventionen außer Kraft gesetzt wurden (S. 14 ff., 47 ff.). Die wichtige koloniale Kontrollfunktion, die das Judicial Committee of the Privy Council als oberstes Rechtsmittelgericht des Commonwealth ausübte, wird herausgestellt.

Das zweite Kapitel setzt sich ausführlich mit zwei Fragen auseinander, die sich aus dem eigentümlichen Auflösungsprozeß des britischen Empire ergeben:

a) Sind die Dominions und alle anderen neuen Commonwealth-Staaten aus diesem Prozeß als souveräne Staaten hervorgegangen? und

b) Wie verträgt sich die im Commonwealth zu beobachtende Staatenpraxis mit der Auffassung von der Staatensukzession, wie sie in der Völkerrechtslehre überwiegend vertreten wird?

Der Verfasser untersucht zunächst, ob in den ehemals abhängigen Gebieten heute „selbständige Normensysteme“ gelten, wobei er Souveränität mit Bodin als „Befehlsunabhängigkeit" versteht (S. 25). Folgt man wie der Autor der überwiegenden

1 Das französische Kolonialreich und die Gründung neuer Staaten, besprochen in VRU 1970, S. $111 \mathrm{ff}$. 
Auffassung in der britischen Verfassungsrechtslehre zum Grundsatz der Parlamentssouveränität (S. 29 ff.), die allerdings zunehmend Kritiker findet, so bestünde nach striktem britischen Recht weiterhin eine Befehlsabhängigkeit nicht nur der Dominions (S. 33), sondern aller ehemaligen Kolonien. Denn nach dieser These können sowohl das Westminster-Statut wie auch alle späteren Unabhängigkeitsgesetze vom britischen Parlament rechtmäßig aufgehoben werden, lediglich Verfassungskonvention und politische Realität verhindern es. Die praktische Relevanz der Kontroverse ist allerdings nicht sehr hoch $z u$ veranschlagen, denn der Verfasser weist zu Recht darauf hin, daß die Parlamentssouveränität als Regel des britischen Verfassungsrechts in ihrem Geltungsbereich vom völkerrechtlichen Effektivitätsgrundsatz eingegrenzt wird, auch wenn der britische Dualismus daraus für das Verfassungsprinzip selbst noch keine Konsequenzen zieht (S. 42 f.).

Bei der Beantwortung der zweiten Frage versucht der Autor aus der Commonwealth-Praxis Schlußfolgerungen für die allgemeine Lehre von der Staatensukzession zu ziehen (S. 72 ff.). Bezüglich der materiellen Rechtsfortgeltung ergibt sich aus den Unabhängigkeitsgesetzen für die ehemals britischen Kolonien, daß grundsätzlich von der Kontinuität der gesamten Rechtsordnung auszugehen ist (S. 74 f.). Selbst, wo die Unabhängigkeitsverfassung das alte Verfassungsrecht ändert, ist sie in einer britischen Order in Council enthalten, so daß die materielle Diskontinuität zumindest durch formelle Kontinuität vermittelt wird (S. 84). Auch wenn man nicht mit dem Verfasser der Auffassung ist, diese Commonwealth-Praxis führe zu einer Widerlegung der herrschenden Sukzessionslehre im allgemeinen (ebda.), so ist die Annahme einer materiellen, zumindest aber formellen Rechtskontinuität im Commonwealth aus folgendem Grund überzeugend: die Briten haben ihre Kolonien mit einer bestimmten, meist nur erschwert abänderbaren Verfassung in die Unabhängigkeit entlassen, um die politische Entwicklung in den jungen Staaten auch über den Unabhängigkeitstag hinaus bis $\mathrm{zu}$ einem gewissen Grade zu determinieren. Bei einer Diskontinuität der Rechtsordnung wäre dieser Versuch auf etwas rechtlich Unmögliches gerichtet gewesen (S. 82, 88). Die Fortdauer des Geltungsgrundes ist nach der richtigen Auffassung des Autors der Souveränität des Nachfolgerstaates ebensowenig abträglich (S. 85), wie der Umstand, daß nach britischem Staatsrecht das Parlament in Westminster jederzeit die Unabhängigkeitsverfassung dieses Staates aufheben kann.

Abschließend wird im vierten Kapitel kurz das Problem der ,Autochthonisierung' erörtert (S. 100 ff.). Damit bezeichnet der Verfasser den bewußten Abbruch der rechtlichen Kontinuitätskette in einigen Commonwealth-Staaten. Die Untersuchung endet mit einer nützlichen Gegenüberstellung derartiger ,Abnabelungsversuche' in Indien und Pakistan und ihrer unterschiedlichen richterlichen Bewertung (S. 110 ff.). Hier fehlt noch die grundlegende Entscheidung des pakistanischen Supreme Court in ,Asma Jilani v. Government of the Punjab’2, wo das Gericht von seiner in ,State v. Dosso' aufgestellten Diskontinuitätsthese abrückte und die Rechtskontinuität nach einem weiteren Staatsstreich religiös $z u$ begründen suchte.

$\mathrm{Da}$ der Autor zuvor festgestellt hat, daß die Souveränität der jungen Staaten nicht von einem autochthonen Geltungsgrund abhängt, läß sich das Streben nach Autochthonie nur als juristisch überspitzte Form nationalistischer

2 P. L. D. 1972 S. C., S. 139 ff. 
Politik erklären. Hier wird deutlich, welchen beschränkten Erklärungswert ein Begriff wie ,Autochthonie' hat, wenn er so eng gefaßt wird wie in dieser Untersuchung. Yash P. Ghai hat an anderer Stelle ${ }^{3}$ diese Begriffsbildung kritisiert, die die Erörterung von vornherein auf die rechtstechnische Unterbrechung der verfassungsrechtlichen Kontinuitätskette begrenzt und materielle Entwicklungen des Verfassungsrechts der neuen Staaten ausklammert. So will es z. B. nicht einleuchten, daß Tansanias Verfassung nur „auf niederer Stufe autochthon“ ist (S. 110), obwohl dieser Staat sich wohl wie kaum ein anderes Commonwealth-Mitglied in seiner internen Struktur vom ,Westminster export model' entfernt und sein Verfassungsrecht damit in einem weiteren Sinne, autochthonisiert' hat. Hier wird deutlich, daß der Abbruch der Kontinuitätskette nur ein denkbares - vielleicht gar nicht das wirksamste - Mittel zur Autochthonisierung der Rechtsordnung ist. Sicherlich lag eine solche Autochthonie im weiteren Sinne außerhalb des Rahmens, den der Verfasser für seine hier besprochene Untersuchung gesteckt hatte. Es bleibt jedoch zu hoffen, daß die angedeutete Problematik bald zum Gegenstand einer eigenen Analyse gemacht wird, um einen umfassenden Überblick über die rechtlichen Aspekte und Konsequenzen der britischen Dekolonisationspolitik zu vermitteln. Der Verfasser hat mit seinem Buch einen recht speziellen, darum aber nicht weniger wertvollen Beitrag zur Beschreibung der juristischen Entkolonialisierung im Commonwealth geleistet.

Alexander Dix

\section{KI-Whan NAM}

Völkerrechtliche und staatsrechtliche Probleme des zweigeteilten Korea und die Frage der Vereinigung der koreanischen Nation

Europäische Hochschulschriften - Rechtswissenschaft Bd. 113, Herbert Lang, Bern und Peter Lang, Frankfurt/M., 1975, 239 S., sFr. 43, 20

Es war am 4. Juli 1972, als nicht nur Koreaner, sondern viele andere Völker in der Welt das sogenannte „Nord- und Südabkommen“ begeistert aufnahmen. In diesem Abkommen vereinbarte Korea die Wiedervereinigung des Landes durch „unabhängige koreanische Bestrebungen auf friedlichem Wege und auf der Grundlage des ursprünglich homogenen Volkes". Aber die koreanische Bevölkerung ist von der gegenwärtigen feindseligen politischen Entwicklung zwischen Nord- und Südkorea so enttäuscht, daß sie das „wirkliche Ziel“, den koreanischen Dialog, seit 1971 anzweifelt ${ }^{1}$. Deshalb untersuchen z. Z. nicht nur $\mathrm{ich}^{2}$, sondern viele Wissenschaftler, wie z. B. Gregory Henderson, Harry Sichrovsky und Joungwon A. $\mathrm{Kim}^{3}$, den Grund des „erfolglosen“ koreanischen Dialoges und die Möglichkeit der Wiedervereinigung Koreas. In diesem Sinne gratuliere ich Herrn Ki-Whan Nam für seine Promotion mit dieser Dissertation herzlich.

Das erste Kapitel (S. 8-61) handelt über die völkerrechtliche Anerkennung des Staates. Als theoretischen Teil faßt der Verfasser die Voraussetzungen der Pflicht

3 ICLQ 1973, S. 403 ff., 414 f.

1 Vgl. Youn-Soo Kim: Korea - Die staatsrechtliche Problematik eines geteilten Staates im Vergleich zur Bundesrepublik Deutschland. In: VRƯ, 4. Heft 1974, S. 427-441

2 Youn-Soo Kim: Die Teilung und Wiedervereinigung Koreas 1945-1973 - Analyse und Dokumente, Kiel 1973

3 Gregory Henderson: Divided Nation in a Divided World. New York 1974; Harry Sichrovsky: Korea Report - Vom Bruderkrieg zur Wiedervereinigung? Wien 1973; Joungwon A. Kim: Divided Korea The Politics of Development 1945-1972. Cambridge/Massachusetts 1975 